\title{
Heparin versus Saline Solution for Locking of Totally Implantable Venous Access Port (TIVAP): Cohort Study of the First Kurdistan Series of TIVAP
}

\author{
Aram Baram 1*, Goran Majeed1, Hazha Abdullah ${ }^{2,3}$, Allaa Subhi1 \\ ${ }^{1}$ Department of Thoracic and Cardiovascular Surgery, Faculty of Medical Sciences, School of Medicine, \\ University of Sulaimani, Sulaymaniyah, Iraq \\ ${ }^{2}$ Department of Medicine, Faculty of Medical Sciences, School of Medicine, University of Sulaimani, \\ Sulaymaniyah, Iraq \\ ${ }^{3}$ Hewa Center for Medical Oncology, Sulaymaniyah, Iraq \\ Email: *aram.baramm@gmail.com, goransalah.83@gmail.com, hazhaabdulla@yahoo.com \\ allaassubhi@yahoo.com
}

Received 10 September 2014; revised 20 October 2014; accepted 5 November 2014

Copyright (C) 2014 by authors and Scientific Research Publishing Inc.

This work is licensed under the Creative Commons Attribution International License (CC BY). http://creativecommons.org/licenses/by/4.0/

(c) (i) Open Access

\begin{abstract}
Introduction: Totally implantable venous access port (TIVAP) is essential prerequisite for most of chemotherapy protocols. Flushing with $0.9 \%$ sodium chloride becomes an alternative to heparinnized solution. As flushing and locking solutions are still controversial, this study was conducted to compare efficacy of heparinized solution versus normal saline solution for locking in ports TIVAP. Patients and Methods: Prospective Cohort study performed in teaching hospital Sulaymaniyah-University of Kurdistan, Iraq, including 384 TIVAP implanted in cancer and non-cancer patients. The study reports the TIVAP outcome in 2 groups of patients where 2 different solutions used for maintaining catheter's patency by heparinized solution in group (A), versus normal saline for group (B). Results: In group A, the rate of complications was $8.2 \%(n=16)$ while in group B complications rate was $7.9 \%(n=15)$. Thrombosis in group $A$ occurred in $1.03 \%$ of the cases and in group B was $1.57 \%$. There were no significant differences between the two groups regarding the causes for unwanted removals of the TIVAP. Conclusions: The results of our study suggest that heparin has no role in preventing the early or late complications of TIVAP and we do not recommend using it as a locking solution.
\end{abstract}

\section{Keywords}

TIVAP, Locking Solution, Port Thrombosis, Surgical Site Infection, Kurdistan-Iraq

\footnotetext{
${ }^{*}$ Corresponding author.
}

How to cite this paper: Baram, A., Majeed, G., Abdullah, H. and Subhi, A. (2014) Heparin versus Saline Solution for Locking of Totally Implantable Venous Access Port (TIVAP): Cohort Study of the First Kurdistan Series of TIVAP. Advances in Lung Cancer, 3, 67-74. http://dx.doi.org/10.4236/alc.2014.34010 


\section{Introduction}

Currently, totally implantable venous access port (TIVAP) becomes an essential prerequisite for most of chemotherapy protocols for solid and hematological malignancies and chronic illnesses require long duration care [1]. TIVAP is a subcutaneously implanted port made of titanium connected to a silicon central venous catheter (Figure 1) which provides a safe and easy long term vascular access for the delivery of chemotherapy and supportive therapy including fluid, blood products, solutions for parenteral nutrition and intravenous medications [1]. In addition, it facilitates frequent blood sampling with minimal discomfort [2].

The drawback of using TIVAP is the occurrence of the unexpected and undesirable complications. Although, the risk of infection with ports occurs less frequently than with catheters [3], complications such as infection, thrombosis, and catheter fracture are still a matter of concern, which may have adverse effect on the patient and may require undesirable removal of the port [4].

Flushing and locking TIVAPs with anticoagulant solutions in the intervals between infusions are often included in the maintenance policy to prevent occlusion, although controversy exists over the most appropriate and safe approach [3] [5].

Flushing and locking of the TIVAP with unfractionated heparin solution is used to be routinely recommended to prevent its obstruction [6]. Moreover, though rare, heparin may cause drug interaction, heparin induced thrombocytopenia and hemorrhage [6], thence controversies regarding the efficacy of this practice recently been a matter of debate between many health institutions around the world [3] [5] [6].

As consequence of these debates, flushing with $0.9 \%$ sodium chloride or normal saline alone started to be used as an alternative to heparinized solution.

In this prospective cohort study we report our results for the first patient series with TIVAP in Iraq and as flushing and locking solutions are still controversial, this study was conducted to compare efficacy of heparinnized solution versus normal saline solution for locking purposes in ports of cancer and non-cancer patients.

\section{Patients and Methods}

A prospective comparative study was carried out at the teaching hospital of Sulaymaniyah partner the university of Sulaimani-Kurdistan/Iraq, including 384 totally Implantable Venous Access Port (TIVAP) implanted in cancer and non-cancer patients from January 2010 to July 2013. The study reports the (TIVAP) outcome observed in 2 groups of patients where 2 different solutions used for maintaining catheter's patency, that is, by using heparinized solution in group (A), versus using normal saline solution only for locking the device in the period between infusions in group (B). Although during the entire duration of the study both groups were observed for any local or systemic complications and all were documented. All patients were followed for a minimum period of 24 months and data collection terminated as of July $31^{\text {st }} 2013$. Clinical patients' data and data on the duration and reason for failure of the ports were extracted from outpatient clinic records. The medical records of each patient were prospectively documented to obtain the following information: Baseline clinical patient data, date of implantation and removal of the port, and cause of removal this was classified in 4 groups: irreversible occlusion (the primary study end-point), other complications, end of treatment, or patient's death.

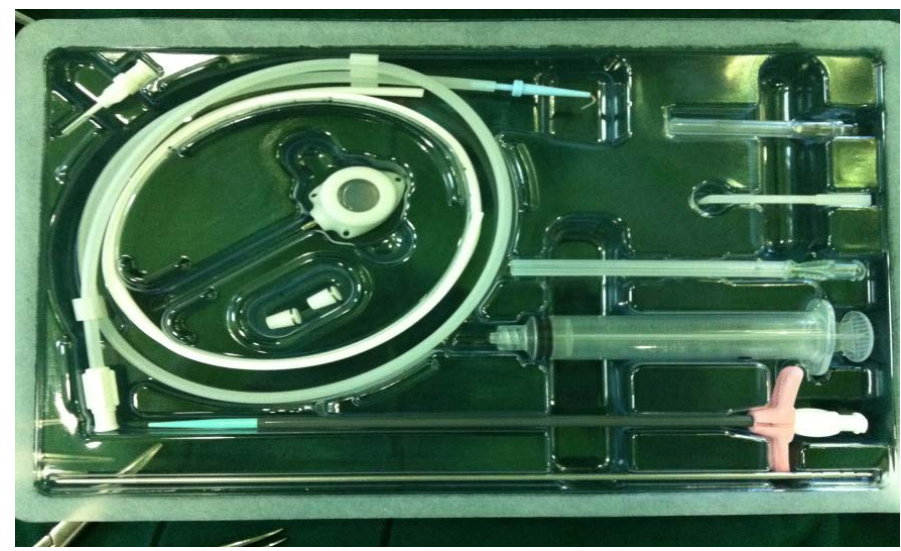

Figure 1. TIVAP with its insertion kit. 


\subsection{Study Design}

This prospective comparative study compares the outcome of (TIVAP) in 2 consecutive groups of patients. The patients were distributed to either group randomly. We used the same technique of insertion for all and the same regime of care been offered for both. The 2 policies for locking ports were consistently used in all patients within each of 2 different consecutive periods, the remainder of a port management being similar in either group.

\subsection{Objectives}

1) To evaluate the efficacy and safety of normal saline solution for intermittent locking practice of implanted ports in cancer and non-cancer patients.

2) To report any complications during follow-up.

3) To report the first series of patient having TIVAP inserted in Kurdistan/Iraq.

\subsection{Exclusion Criteria}

1) All patients who had platelet count below 30000 per cubic millimeter and it was not correctable was excluded from this study.

2) Local skin infection near to the presumable site of implantation

\subsection{Implantation Technique}

The same brand device (POLYSITE ${ }^{\circledR} 4000$ series-Hybrid form PEROUSE MEDICAL-France) (Figure 1, Figure 2) was implanted in both groups. Majority of the devices (97\%) were inserted under local anesthesia, only 12 devices (3\%) implanted under general anesthesia because of inability to be inserted under local anesthesia in children. Subclavian vein used in majority but only 3 ports inserted in the femoral vein because subclavian and internal jugular vein thrombosis in these patients. For the Subclavian approach a $4 \mathrm{~cm}$ incision performed (after disinfecting the operative site by Povidone-iodine 10\%) at $2 \mathrm{~cm}$ below the mid third of the clavicle followed by creating a $4 * 4 \mathrm{~cm}$ pocket for the port subcutaneously (Figure 3). Seldinger's technique used to introduce the catheter to the vein, catheter proper positioning verified by fluoroscopy (Figures 4(a)-(c)). The same technique



Figure 2. Graded catheter.

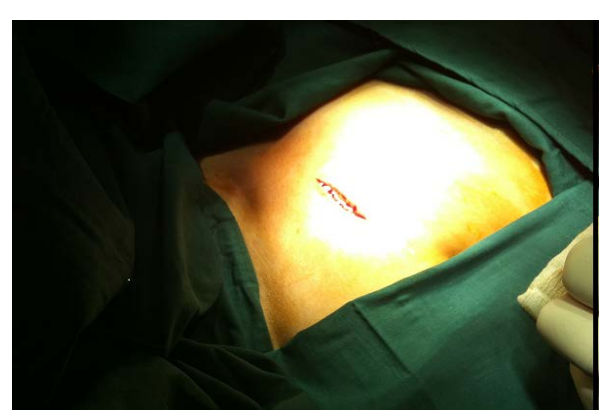

Figure 3. Subcutaneous pocket $4 * 4 \mathrm{~cm}$ prepared for the port insertion. 


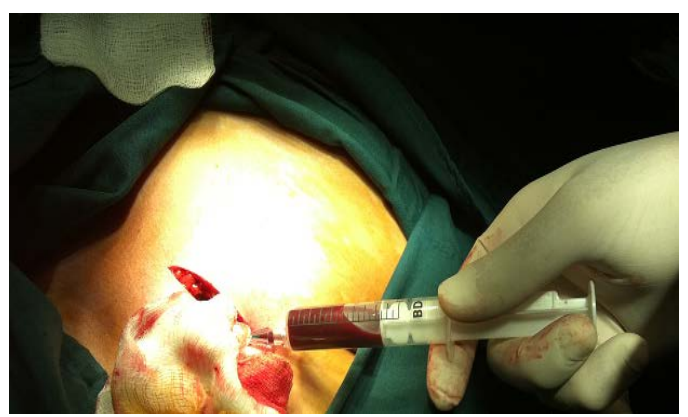

(a)

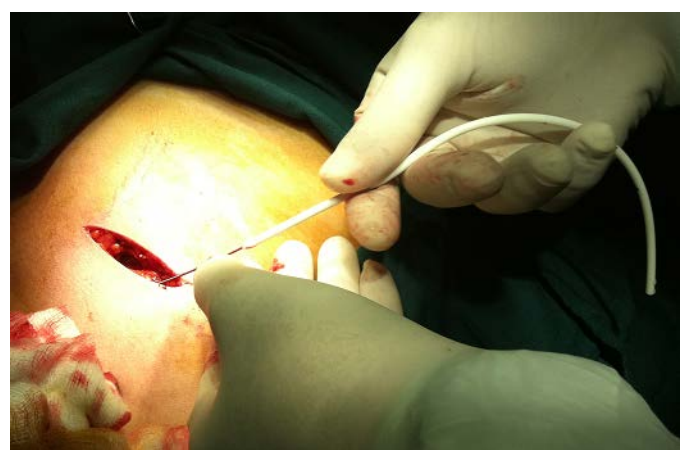

(c)



(b)

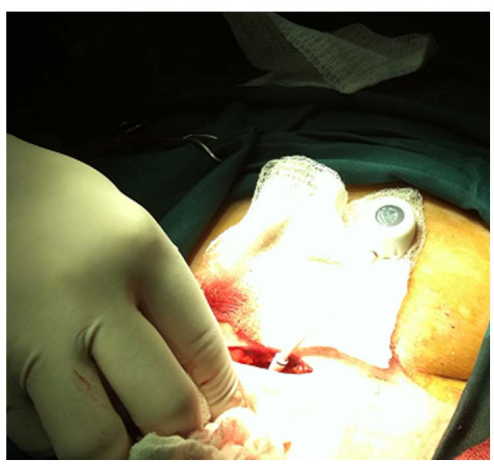

(d)

Figure 4. (a) Guide wire insertion to the right atrium insertion verified by fluoroscopy; (b) Catheter insertion over the guide wire; (c) Port attached to the catheter before final implantation; (d) Port attached to the catheter before final implantation.

used for femoral vein approach, the incision was performed at $2 \mathrm{~cm}$ below the midpoint of the inguinal ligament and the port pocket was done in the lower abdomen. All patients received single dose (according to the weight) of third generation cephalosporin as a prophylactic and whenever signs of infection detected this was continued until total resolution of the symptoms.

\subsection{TIVAP Care}

All consecutive 384 (TIVAP) device implantation in cancer and non-cancer patients at Sulaymaniyah teaching hospital during the study period were include. All patients were outpatients receiving chemotherapy, coagulation factors or parenteral nutrition at fixed intervals according to protocol schedules for different types of tumors or their hematological disorders. In group A ( $n=194)$ TIVP locked twice monthly with heparinized solution (20 $\mathrm{mL}$ normal saline and 5000 international unit of unfractionated heparin). This group of patients was assumed as the control group. In group B $(n=190)$ ports were locked twice monthly with $20 \mathrm{~mL}$ of normal saline solution. Locking procedure was carried out under aseptic technique by a pulsatile method with the use of a 20 -mL syringe and a non-coring Huber's needle. Any abnormality in the flushing technique during the procedure was recorded.

\section{Study Endpoint}

1. The primary study endpoint, irreversible occlusion, was defined by the failure of 2 different consecutive attempts to restore catheter patency.

2. Completion of the treatment

3. Symptoms and signs of infection necessitate removal of the port

\section{Results}

During a 42-month period, 384 consecutive adult and pediatric cancer and non-cancer patients were underwent 
TIVAP implantation in our unit; their characteristics are shown in Table 1. In group A, during the period of follow up ranged 6 - 30 months (mean 15.6 months) the rate of complications was 8.2\% $(\mathrm{n}=16)$ while during the same period in group B complications rate was $7.9 \%(n=15)$ as shown in Table 2. Among these complications of particular concern is thrombosis of the port and catheter, in group A thrombosis occurred in $1.03 \%$ of the cases while in group B was $1.57 \%$ only, the rate of other complications shown for the two groups comparatively in Table 3. The rate of complications was not significant statistically $(p=0.89)$.

The causes of unwanted removals of the TIVAP was reviewed in the two groups which showed no significant difference for all causes, in group A thrombosis occurred in $2(1.03 \%)(p=0.605)$ patients while only $3(1.57 \%)$ $(p=0.805)$ patients had this complication in group B. No significant differences were noticed for other complications as demonstrated in Table 4. In group A totally 9 patients (4.6\%) developed surgical site infection, 4 (2.05\%) of these caused unwanted removal and 5 patients treated by appropriate antibiotic therapy. SSI occurred in $6(7.7 \%)$ patients of group B from which 3 caused unwanted loss of the device. No mortality reported due to TIVAP implantation in either group.

Table 1. Patient's characteristics in both groups.

\begin{tabular}{|c|c|c|c|c|c|c|}
\hline \multirow{2}{*}{ Characteristics } & \multicolumn{2}{|c|}{ Group A $(n=194)$} & \multicolumn{2}{|c|}{ Group B (n = 190) } & \multicolumn{2}{|c|}{ Total $(n=384)$} \\
\hline & $\mathbf{N}$ & $\%$ & $\mathbf{N}$ & $\%$ & $\mathbf{N}$ & $\%$ \\
\hline \multicolumn{7}{|l|}{ Age } \\
\hline$<50$ & 110 & 56.7 & 80 & 42.1 & 190 & 49.5 \\
\hline$\geq 50$ & 84 & 43.3 & 110 & 57.9 & 194 & 50.5 \\
\hline \multicolumn{7}{|l|}{ Sex } \\
\hline Male & 62 & 31.9 & 74 & 38.9 & 136 & 35.4 \\
\hline Female & 132 & 68.1 & 116 & 61.1 & 248 & 64.6 \\
\hline \multicolumn{7}{|l|}{ Underline disease } \\
\hline Leukemia/Lymphoma & 34 & 18.5 & 24 & 12.6 & 58 & 15.1 \\
\hline Solid tumor/others & 160 & 82.5 & 166 & 87.4 & 326 & 84.9 \\
\hline \multicolumn{7}{|l|}{ French diameter } \\
\hline$<8 \mathrm{~F}$ & 36 & 18.5 & 18 & 9.5 & 54 & 14 \\
\hline$\geq 8 \mathrm{~F}$ & 158 & 81.5 & 172 & 90.5 & 330 & 86 \\
\hline \multicolumn{7}{|l|}{ Accessed vein } \\
\hline Subclavian vein & 194 & 100 & 184 & 96.8 & 378 & 98.4 \\
\hline - Right & 148 & 76.3 & 138 & 72.6 & 286 & 74.5 \\
\hline - Left & 46 & 23.7 & 46 & 24.3 & 92 & 24 \\
\hline Femoral vein & 0 & 0 & 6 & 3.2 & 6 & 1.6 \\
\hline - $\quad$ Right & 0 & 0 & 4 & 2.1 & 4 & 1 \\
\hline - Left & 0 & 0 & 2 & 1 & 2 & 0.5 \\
\hline \multicolumn{7}{|l|}{ Type of anesthesia } \\
\hline LA & 182 & 93.8 & 186 & 97.9 & 368 & 95.8 \\
\hline GA & 12 & 6.2 & 4 & 2.1 & 16 & 4.2 \\
\hline
\end{tabular}

Table 2. Two group comparison.

\begin{tabular}{ccc}
\hline Complications & Group A (HS) & Group B (NS) \\
Present & $16(8.2 \%)$ & $15(7.9 \%)$ \\
Not Present & $178(91.7 \%)$ & $175)$ \\
Total no. of cases & 194 & 190 \\
\hline
\end{tabular}

Note: $p=0.89$. 
Table 3. Rate of complications.

\begin{tabular}{|c|c|c|c|c|c|c|}
\hline \multirow{2}{*}{ Complications } & \multicolumn{2}{|c|}{ Group A (HS) } & \multicolumn{2}{|c|}{ Group B (NS) } & \multicolumn{2}{|c|}{ Total } \\
\hline & $\%$ & No. & $\%$ & No. & $\%$ & No. \\
\hline Surgical site infection (SSI) & 4.63 & 9 & 3.1 & 6 & 7.73 & 15 \\
\hline Thrombosis & 1.03 & 2 & 1.57 & 3 & 2.6 & 5 \\
\hline Venous thrombosis & 1.03 & 2 & 1.05 & 2 & 2.08 & 4 \\
\hline Hematoma & 0.51 & 1 & 0 & 0 & 0.51 & 1 \\
\hline Catheter fracture & 0.51 & 1 & 0 & 0 & 0.51 & 1 \\
\hline Pneumothorax & 0.51 & 1 & 0 & 0 & 0.51 & 1 \\
\hline Catheter migration & 0 & 0 & 1.05 & 2 & 1.05 & 2 \\
\hline Right upper limb pain & 0 & 0 & 0.52 & 1 & 0.52 & 1 \\
\hline Right ventricular placement & 0 & 0 & 0.52 & 1 & 0.52 & 1 \\
\hline Total & 8.22 & 16 & 7.9 & 15 & 16.03 & 31 \\
\hline
\end{tabular}

Note: Group A: $p=0.605$; Group B: $p=0.805$.

Table 4. Causes of unwanted removal during the period of the follow up.

\begin{tabular}{ccccc}
\hline & \multicolumn{3}{c}{ Group A (HS) } & \multicolumn{2}{c}{ Group B (NS) } \\
\cline { 2 - 5 } Cause of removal of the TIVAP & $\%$ & No. & \% & No. \\
\hline Surgical site infection & 2.05 & 4 & 1.55 & 3 \\
Thrombosis & 1.03 & 2 & 1.57 & 0 \\
Catheter fracture & 0.51 & 1 & 0 & 0 \\
Catheter migration & 0.51 & 1 & 1.05 & 2 \\
\hline Right ventricular placement & 0 & 0 & & 0 \\
\hline
\end{tabular}

\section{Discussion}

Patency of TIVAP is a common problem in all health care settings. Lack of lumen patency disrupts patient care, threatens achievement of treatment goals, adds to the burden of limited nursing resources and increases cost of care. TIVAP that do not produce a brisk blood return on aspiration are considered to be nonfunctioning catheters and require further assessment to ensure that the fluid or medications will not leak into extra-vascular [3] [7]. Catheters occlude due to multiple reasons; however, thrombotic occlusion is the most common cause [4].

To maintain its patency, it is essential for TIVAP to be flushed intermittently. Although it is controversial, flushing and locking with heparin saline solution has become a stander protocol which has been practiced for many years to prevent occlusion of vascular lines [3] [6] [8].

In the recent years, several issues regarding the use of heparin as a locking solution have been raised. Firstly, heparin is an anticoagulant not a thrombolytic agent, by which, it does not help thrombolysis but only prevent further progression of the existing clot. In addition, it has a short half-life (90 minutes) [9].

Secondly, spillage of heparin in to the circulation may cause heparin-induced thrombocytopenia [8] [9]. This is a serious complication especially for those who have blood malignancies and they already suffer from low platelet count and are on a high risk to develop bleeding [4] [6] [8] [10]. Moreover, there are drugs that are incompatible with heparin; injecting them through a port locked with heprinized solution may create problems [11] [12].

In our study, the incidence of the overall complication (the two groups collectively) was $16.03 \%$. This percentage is nearly similar to some of the studies that have been carried out to assess the complications of TIVAP 
[13]-[15]. Surgical site infection (SSI), Thrombosis, Haematoma, Catheter Fracture, Catheter migration, Right upper limb pain, Pneumothorax and Right ventricular placement are the complications that have been recorded in this study with frequencies similar to other papers [13]-[15]. Apart from SSI, there was no statistically significant difference of the other complications between the two groups (Table 3).

SSI was the only significantly different complication which was higher in group A $4.63 \%$ versus $3.1 \%$ in group B. No explanation for difference in SSI between the 2 groups was found.

Maintaining catheter patency is the main issue that encourages the use of heparin. In this study heparin showed no obvious effect in solving this problem, as thrombosis was not significantly different as in group A thrombosis occurred in $2(1.03 \%)$ patients while only $3(1.57 \%)$ patients had this complication in group B. The incidence of catheter-related venous thrombosis was quite low in this study (2.6\%) for both groups collectively, with no significant differences among the two groups. The association between cancer and thrombosis arises as a consequence of cancer treatment and direct vessel trauma, as a result of long-term central venous catheter placement [18]. Considering all the side effects of heparin discussed above and with the results that we found in this study about its efficacy in preventing thrombosis of the TIVAP and the harboring vein we think heparin is not an effective substance in preventing these thrombotic complications. Similar results were reported by different authors [3] [16] [17] [19].

Our two groups of patients are heterogeneous samples contains adults, pediatric, young and old age patients having cancer and non-cancer pathologies, but despite that variety we found no any difference in the efficacy of heparin locking solution in preventing thrombotic complications.

Majority of venous access sites in our study was subclavian vein (group A $100 \%$ vs. $96 \%$ in group B) with relatively very low rate of puncture site hematoma in one patient $(n=1)$, pneumothorax in another patient. No any arterial puncture reported despite that we did not used any sonographic guide to localize the vein, these findings is are not agreed by other investigators who reported that the use of sonographic guidance may decrease the rate of puncture related complications [20]-[23].

Insertion of the TIVAP was done under local anesthesia in 93\% of the cases, general anesthesia was used only for pediatric population, all the implantations are performed in the vascular surgery suite this may be the possible explanation for the lower rate for all type of complications as many papers showed the higher rate of complication for TIVAP insertion in radiology suite insertion [24] [25].

\section{Conclusion}

The results of our study suggest that heparin has no role in preventing the early or late complications of TIVAP and we do not recommend using it as a locking solution. Implantation of TIVAP by vascular surgeons has better outcome in comparison to the interventional radiologist procedures.

\section{Conflict of Interest}

The authors declare that they have no financial or personal relationships with other people or organizations that could have inappropriately influenced or biased their work.

\section{Acknowledgements}

We would like to thank all our paramedical staffs who assisted in the care of the implanted ports. Our special thanks and gratitude to Dr. Laith Abdul Majeed, Dr. Saywan Kaka Rash for their assistance in the statistical analysis.

\section{References}

[1] Teichgräber, U.K., Pfitzmann, R. and Hofmann, H.A.F. (2011) Central Venous Port Systems as an Integral Part of Chemotherapy. Deutsches Ärzteblatt International, 108, 147-54.

[2] Olivia, A., et al. (2012) Totally Implantable Central Venous Access Devices in Patients with Cancer: Experience in a Private Oncological Center. Cirugía y Cirujanos, 80, 400-404.

[3] Bertoglio, S., et al. (2012) Efficacy of Normal Saline Versus Heparinized Saline Solution for Locking Catheters of Totally Implantable Long-Term Central Vascular Access Devices in Adult Cancer Patients. Cancer Nursing, 35, E35-E42. http://dx.doi.org/10.1097/NCC.0b013e31823312b1 
[4] Marcy, P.-Y. (2008) Central Venous Access: Techniques and Indications in Oncology. European Radiology, 18, 23332344. http://dx.doi.org/10.1007/s00330-008-0981-2

[5] Bassi, K.K., Giri, A.K., Pattanayak, M., Abraham, S.W. and Pandey, K.K. (2012) Totally Implantable Venous Access Ports: Retrospective Review of Long Term Complications in 81 Patients. Indian Journal of Cancer, 49, 114-118. http://dx.doi.org/10.4103/0019-509X.98934

[6] Fernanda et al. (2008) Patients’ Perception Regarding the Use of a Long-Term Catheter. Revista da Escola de Enfermagem da USP, 42, 518-523.

[7] Krupski, G., Froschle, G.W., Weh, F.J. and Schlosser, G.A. (1995) Central Venous Access Devices in Treatment of Patients with Malignant Tumors: Venous Port, Central Venous Catheter and Hickman Catheter. Cost-Benefit Analysis Based on a Critical Review of the Literature, Personal Experiences with 135 Port Implantations and Patient Attitude. Chirurgie, 66, 202-207.

[8] Infusion Nurses Society (2011) Infusion Nursing Standards of Practice. Journal of Infusion Nursing, 34.

[9] Cesaro, S., et al. (2009) Prospective, Randomized Trial of Two Different Modalities of Flushing Central Venous Catheters in Pediatric Patients with Cancer. Journal of Clinical Oncology, 27, 2059-2065. http://dx.doi.org/10.1200/JCO.2008.19.4860

[10] Michael et al. (2008) Treatment and Prevention of Heparin-Induced Thrombocytopenia: American College of Chest Physicians. Evidence-Based Clinical Practice Guidelines (8th Edition). Chest, 133, 340-380. http://dx.doi.org/10.1378/chest.08-0677

[11] Hadaway, L. (2006) Heparin Locking for Central Venous Catheters. Journal of the Association for Vascular Access, 11, 224-231. http://dx.doi.org/10.2309/java.11-4-17

[12] Zaghal, A., Khalife, M., Mukherji, D., et al. (2012) Update on Totally Implantable Venous Access Devices. Surgical Oncology, 21, 207-215. http://dx.doi.org/10.1016/j.suronc.2012.02.003

[13] Jan, H.-C., Chou, S.-J., Chen, T.-H., et al. (2012) Management and Prevention of Complications of Subcutaneous Intravenous Infusion Port. Surgical Oncology, 21, 7-13. http://dx.doi.org/10.1016/j.suronc.2010.07.001

[14] Araújo, C., Silva, J.P., Antunes, P., et al. (2008) A Comparative Study between Two Central Veins for the Introduction of Totally Implantable Venous Access Devices in 1201 Cancer Patients. European Journal of Surgical Oncology, 34, 222-226. http://dx.doi.org/10.1016/j.ejso.2007.04.003

[15] Rosett, W. and Hodges, G. (1980) Antimicrobial Activity of Heparin. Journal of Clinical Microbiology, 11, 30-34.

[16] LeDuc, K. (1997) Efficacy of Normal Saline Solution versus Heparin Solution for Maintaining Patency of Peripheral Intravenous Catheters in Children. Journal of Emergency Nursing, 23, 306-309. http://dx.doi.org/10.1016/S0099-1767(97)90216-6

[17] Camp-Sorell, D. (2010) State of the Science of Oncology Vascular Access Devices. Seminars in Oncology Nursing, 26, 80-87. http://dx.doi.org/10.1016/j.soncn.2010.02.001

[18] Biffi, R., Orsi, F., Pozzi1, S., Pace, U., et al. (2009) Best Choice of Central Venous Insertion Site for the Prevention of Catheter-Related Complications in Adult Patients Who Need Cancer Therapy: A Randomized Trial. Annals of Oncology, 20, 935-940. http://dx.doi.org/10.1093/annonc/mdn701

[19] Kuter, D.J. (2004) Thrombotic Complications of Central Venous Catheters in Cancer Patients. The Oncologist, 9, 207216. http://dx.doi.org/10.1634/theoncologist.9-2-207

[20] Ahn, S.J., Kim, H.C., Chung, J.W., An, S.B., Yin, Y.H., Jae, H.J. and Park, J.H. (2012) Ultrasound and Fluoroscopy-Guided Placement of Central Venous Ports via Internal Jugular Vein: Retrospective Analysis of 1254 Port Implantations at a Single Center. Korean Journal of Radiology, 13, 314-323. http://dx.doi.org/10.3348/kjr.2012.13.3.314

[21] Karamustafaoglu, Y.A., Yagci, S., Kocal, S. and Yoruk, Y. (2013) Comparison of Implantable Central Venous Ports: Subclavian versus Juguler Access. Journal of Clinical and Analytical Medicine, 4, 495-498. http://dx.doi.org/10.4328/JCAM.1118

[22] Vandoni, R.E., Guerra, A., Sanna, P., Bogen, M., Cavalli, F. and Gertsch, P. (2009) Randomized Comparison of Complications from Three Different Permanent Central Venous Access Systems. Swiss Medical Weekly, 139, 313-316.

[23] Wang, Y.-F., Cherng, S.-C., Chiu, J.-S., et al. (2006) Application of Upper Extremity Radionuclide Venography as a Diagnostic Approach for Port-A Catheter Thrombosis. Journal of the Chinese Medical Association, 69, 358-363. http://dx.doi.org/10.1016/S1726-4901(09)70273-4

[24] Di Carlo, I., Cordio, S., La Greca, G., Privitera, G., Russello, D., Puleo, S. and Latteri, F. (2001) Totally Implantable Venous Access Devices Implanted Surgically: A Retrospective Study on Early and Late Complications. Archives of Surgery, 136, 1050-1053. http://dx.doi.org/10.1001/archsurg.136.9.1050

[25] Stein, M. and Wagner, R.H. (2005) Complications of Central Venous Access Devices Outcome Analysis of 2359 Implantations. Deutsche Medizinische Wochenschrift, 130, 1129-1132. http://dx.doi.org/10.1055/s-2005-866798 
Scientific Research Publishing (SCIRP) is one of the largest Open Access journal publishers. It is currently publishing more than 200 open access, online, peer-reviewed journals covering a wide range of academic disciplines. SCIRP serves the worldwide academic communities and contributes to the progress and application of science with its publication.

Other selected journals from SCIRP are listed as below. Submit your manuscript to us via either submit@scirp.org or Online Submission Portal.
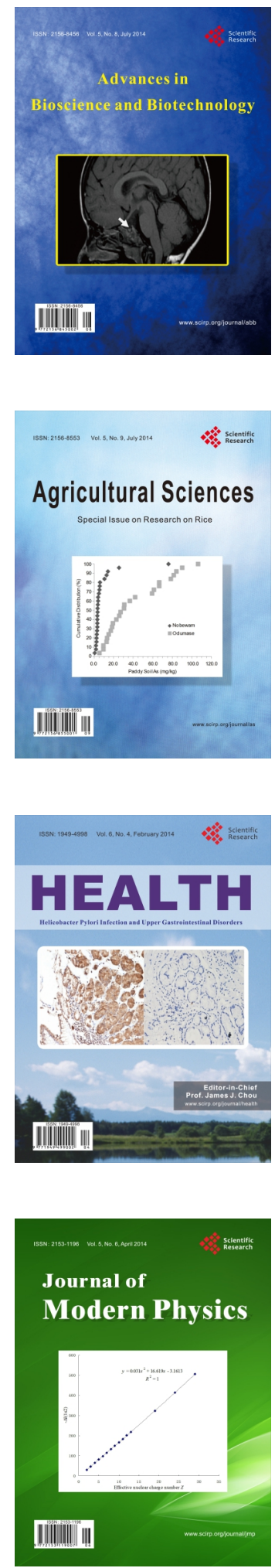
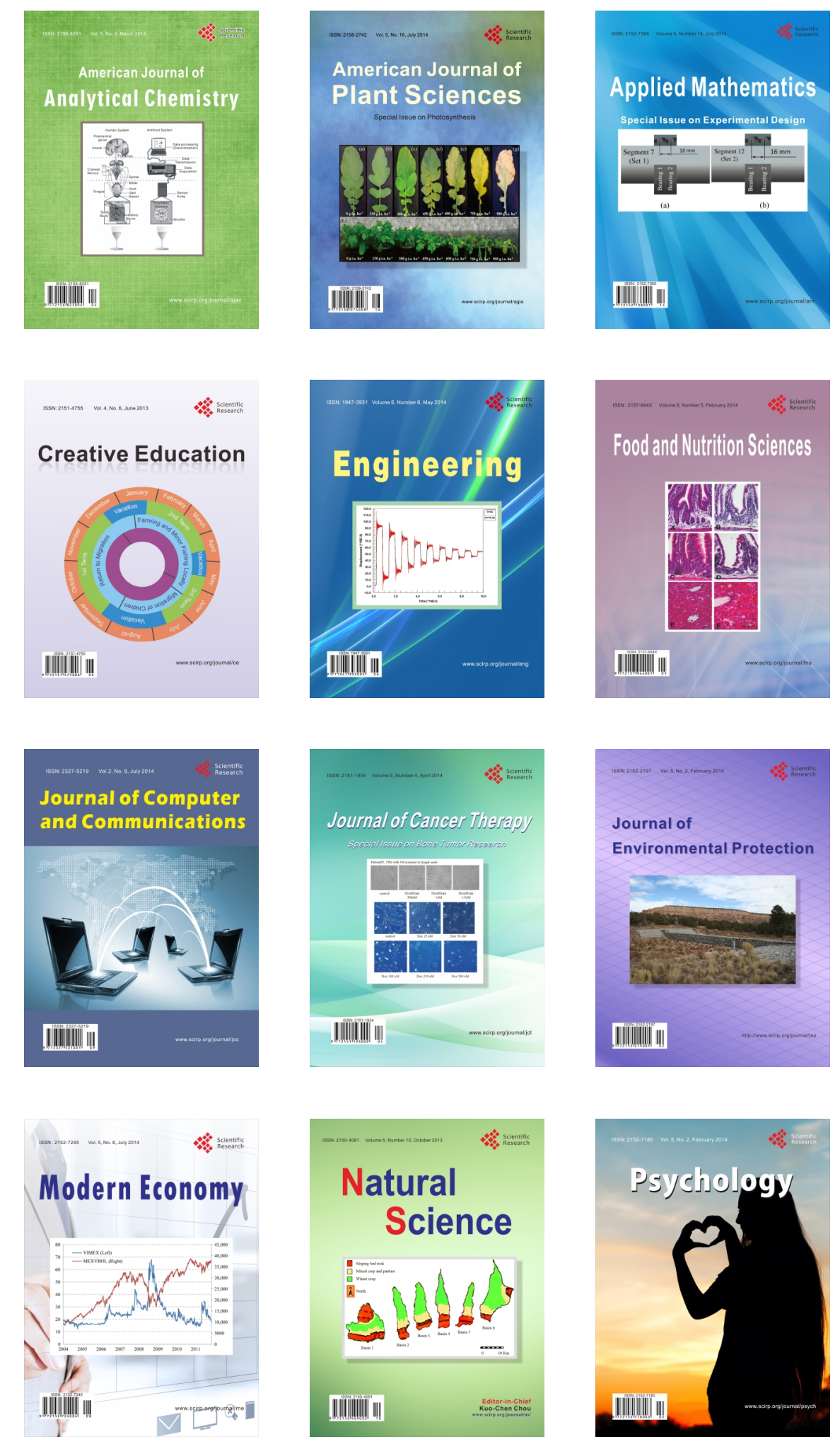